\title{
Vortex shedding in a model of superflow
}

\author{
C. Josserand*, Y. Pomeau \& S. Rica \\ LPS, laboratoire associé au CNRS, Ecole Normale Supérieure, 24, rue Lhomond, 75231 Paris \\ Cedex 05, France \\ and Laboratoire ASCI UPR 9029, Orsay, France.
}

\begin{abstract}
The present article represents part of the $\mathrm{PhD}$. dissertation by C. Josserand $\mathrm{W}$. We discuss the nucleation of quantized vortices in the nonlinear Schrödinger equation (NLS) for a flow around a disk in two spatial dimensions. It appears that the vortices are nucleated when the flow becomes locally (at the edge of the disk) supersonic. A detailed study of the phase equation for the complex field $\psi$ gives an Euler-Tricomi type equation for the stationary solutions below threshold. This equation is closely related to the one known in shock wave dynamics for gas. Then using solvability condition, we extract a time-dependent scenario for the evolution of the amplitude of the solution, which we, finally, relate to a known family solution of NLS which gives rise to a vortex nucleation. We also give a first order correction at the Landau velocity of nucleation, taking into account the geometry of the flow.
\end{abstract}

PACS numbers: 74.20.De; 71.10.-w.

Keywords: Vortex Nucleation, Transonic transition, Superfluidity, Euler-Tricomi equation, Topological vortices.

\section{Contents}

\footnotetext{
*Present Adress: The James Franck Institute, The University of Chicago, 5640 South Ellis Av., Chicago, Illinois 60637, USA.
} 
II $\quad$ Formulation of the problem $\quad 5$

III The Euler-Tricomi equation near the transonic region. $\quad 8$

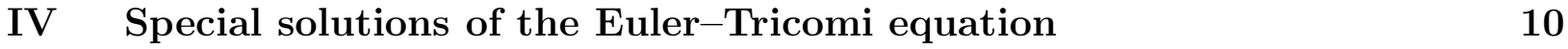

$\nabla \quad$ Regularization of the shock solutions of the Euler-Tricomi equation by $\begin{array}{ll}\text { the quantum pressure. } & 11\end{array}$

\begin{tabular}{lll}
\hline VI & Amplitude equation for the saddle-node bifurcation & 15
\end{tabular}

VII Breakdown of the phase description, the appearance of vortex motion, $\begin{array}{lr}\text { conclusions and further miscellaneous } & 17\end{array}$

\section{INTRODUCTION}

The dynamics of superfluids at zero temperature can be modelled by the Gross-Pitaevskiĩ equation [2]. This is a partial differential equation for a complex valued scalar field $\psi(\mathbf{x}, t)$ with dependence on the position in space, $\mathbf{x}$ and on time $t$. At zero temperature, there is no formal damping term in the equation; it is reversible in time (after complex conjugation) and has even a Lagrangian structure. Although many of properties are well-known and have been studied quite extensively, we shall first review some of them. This Gross-Pitaevskiǐ equation can be seen as a nonlinear Schrödinger equation, and so shares many properties of the linear Schrödinger equation. With periodic boundary conditions, it has an uniform ground state minimizing the energy, the corresponding solution $\psi(\mathbf{x}, t)$ depends periodically on time through a simple exponential factor. As the Gross-Pitaevskir equation is Galilean invariant, it is easy to construct a solution representing an uniform flow by boosting the rest state to a specified speed. With the same model, it is also possible to look at more complicated situations, as flows around obstacles. Two of the authors [3, 4] have studied over the last years a 2D flow around a circular disk (among others), that is the solution of the Gross-Pitaevskiǐ equation with a given uniform and constant flow speed and mass density at 
infinity, with a boundary condition on the surface of the disk. One striking observation has been that beyond a certain critical speed, the flow around the disk becomes time dependent, because vortices are emitted from the disk surface as shown on figure (四).

In Ref. [3] it was shown that the release of vortices from the boundary of the disk is a consequence of a transition from a locally subsonic to supersonic flow. In ordinary fluid mechanics, this would lead to the formation of a shock wave inside the so-called supersonic bubble. Nothing resembling this is possible in the Gross-Pitaevskir equation, because of the lack of built-in irreversibility, something that is necessary to balance nonlinearities inside the shock wave. As argued in Ref. [3], in the present model, the formation of shock waves is replaced -albeit in a rather loose sense- by the nucleation of vortices with a quantized circulation. In fact, these vortices are topological defects solutions of the Gross-Pitaevskiǐ equation. In two space dimensions they are points where the complex field $\psi(\mathbf{x}, t)$ vanishes, making a $\pm 2 \pi$ phase jump when one turns around the defect. These vortices are emitted when the local velocity becomes larger than the critical velocity making the flow locally supersonid

The present work is devoted to study this transition. This is a rather intricate question, as anyone who looked at the paragraph $\S 118, \S 119 \& \S 120$ of Landau and Lifschitz book on fluid mechanics [6] knows well: one has to solve locally the Euler-Tricomi equation for the velocity potential, that can be done in terms of the Airy function. In the present case there is a further complication with respect to Landau's analysis, which comes from the boundary conditions imposed on the disk, see $\S 126$ of Ref. [6]. Let us outline the principles of our analysis; we are dealing with a problem, with two small parameters: the ratio of the microscopic intrinsic length to the disk radius, and the relative shift of the velocity

\footnotetext{
1 The present problem has been studied in the one dimension case by Hakim [5] who has shown that the release of vortices is replaced by a periodic nucleation of one dimensional solitons.
} 
near the pole of the disk to the critical value, a parameter called $\epsilon$.2 Far from the disk boundary, in terms of the microscopic distance, the usual hydrodynamic assumption holds: the quantum pressure term can be neglected, and one obtains an unique, nonlinear equation for the velocity potential's. This equation can be solved by expansion, by assuming that at the dominant order the surface of the disk is flat. In this limit, the hydrodynamic boundary conditions are satisfied with an uniform tangent velocity field. Corrections have to be added to this velocity field in order to take into account the curvature of the disk boundary. The first correction is trivial, and only the second one is crucial for the analysis.

The second order corrections (for the velocity potential) is the solution of the EulerTricomi equation, with specific boundary condition. It happens that the small parameter $\epsilon$ enters into this Euler-Tricomi equation plus the boundary conditions problem in such a way that it can be factored out by rescalings. Moreover, for $\epsilon$ negative, that is for velocities slightly less than the critical speed, the velocity potential at this approximation is multivalued, but only in its extension inside the disk, which is a non-physical part of the flow. At $\epsilon=0$ there is a transition, and the boundary of the region where the velocity potential becomes multivalued enters the physical space, so that the solution of Euler-Tricomi equation cannot be considered as physically acceptable in this range of parameters. In ordinary viscous fluids, this multivaluedness would signal the formation of a shock wave. As said before, no such a thing exists in the present model of superfluid. Accordingly, the shock wave is replaced by a vortex, that is by adding to the flow field in the slightly supersonic region the flow field coming from a localized vortex close to the boundary. Taking in account all the nonlinear terms and the time dependent dynamics on the phase equation, we show that this lack of stationarity appears as the result of a saddle-node bifurcation, as suggested

\footnotetext{
${ }^{2} \epsilon$ is also proportional to the difference between the actual Mach number and its critical value.

${ }^{3}$ In this hydrodynamical limit, the microscopic length scale does not appear, and there is only one small parameter, $\epsilon$.
} 
by two of us some ago 4 . We argue also that the critical velocity behaves as $\sqrt{\frac{\xi_{0}}{R}}$ above the transonic region, being $\xi_{0}$ the healing length of NLS and $R$ the radius of the disk; this is because the quantum pressure stabilizes the flow above the transonic transition for such a range of velocity. We claim that this saddle-node bifurcation, giving rise to time-dependent dynamic corresponds to vortex emission; for that, it remains to match this outer velocity potential, solution of the Euler-Tricomi equation with an inner solution, close to the disk boundary with a vortex.

As one might expect from this rather lengthy introduction, it is a rather uneasy job to put together all this picture, particularly because it depends on properties of solutions the Euler-Tricomi equation that are not obvious. In section 【I, we shall present the general problem in its explicit form, that is the form of the equations as well as the boundary conditions and the relevant facts about the hydrodynamic limit. We finally (section III) obtain a nonlinear equation for an additional phase which can be decomposed as the usual Euler-Tricomi equation plus nonlinear terms that will be treated as perturbations. In section [I], we study some properties of the Euler-Tricomi equation, particularly we point out the multivaluedness solutions of such an equation. In section $\nabla$, we solve the Euler-Tricomi equation (including the quantum pressure term) using a Fourier transform along the $x$-axis first and finding the solution in term of the Airy function. This gives a first correction to the critical velocity coming from the interplay between supersonic flow and regularisation due to the quantum pressure. Then, in section V1, we are able to compute solvability conditions around this critical value of $\epsilon$ that lead to time dependent amplitude equation. These amplitude equations describe a saddle-node bifurcation. In conclusion, in section VII, we try to match this phase approach, which formally cannot describe vortices in NLS, with the release of vortices observed in simulation using the full nonlinear Schrödinger equation.

\section{FORMULATION OF THE PROBLEM}

The Gross-Pitaevskiǐ equation reads, in a dimensionless form: 


$$
i \partial_{t} \psi(\mathbf{x}, t)=-\frac{1}{2} \nabla^{2} \psi+\psi(\mathbf{x}, t)|\psi(\mathbf{x}, t)|^{2}
$$

This is a partial differential equation for a complex wave function $\psi(\mathbf{x}, t)$. This equation is among other properties conservative and Hamiltonian. The ground state in a periodic box is the homogeneous solution: $\psi_{0}=\sqrt{\rho_{0}} e^{-i \rho_{0} t}$. Long wavelength and low amplitude perturbations propagate with the sound speed $c_{s}=\sqrt{\rho_{0}}$, on the other hand; $\xi_{0} \sim \frac{1}{\sqrt{\rho_{0}}}=\frac{1}{c_{s}}$ is the only characteristic microscopic length contained in this equation.

Writing $\psi=\rho^{1 / 2} e^{i \phi}$, we obtain two "real" hydrodynamical fields, $\rho$ and $\phi$ representing respectively the particle fluid density and the velocity potential:

$$
\begin{aligned}
\partial_{t} \rho & =-\nabla \cdot(\rho \nabla \phi) ; \\
\partial_{t} \phi & =-\frac{1}{2}(\nabla \phi)^{2}+\frac{1}{2 \rho^{1 / 2}} \nabla^{2} \rho^{1 / 2}-\rho .
\end{aligned}
$$

The first one is the density mass conservation equation, identifying $\nabla \phi$ by the local velocity $\mathbf{v}$. In the second equation, the term $\frac{1}{2 \rho^{1 / 2}} \nabla^{2} \rho^{1 / 2}$, often called quantum pressure is negligible for large scale flows, that is for flows with a space scale much larger than the intrinsic microscopic length, $\xi_{0}$. When this quantum pressure is neglected, the equation for $\phi$ is the equivalent of Bernoulli's equation for a compressible fluid, with an equation of state for the pressure $(p): p=\frac{1}{2} \rho^{2}$. For a stationary flow, $\partial_{t} \phi$ is a constant, defined by the conditions at infinity. Therefore, the mass density $\rho$ can be computed everywhere as a function of $v$, the modulus of the velocity from this Bernoulli equation.

¿From equations (2) and (3), we obtain the equations for the stationary flow around a disk (of radius $R$, much bigger than any intrinsic length scale $\xi_{0}$ ) with a velocity at infinity $v_{\infty}:$

$$
\begin{aligned}
\nabla \cdot(\rho(|\nabla \phi|) \nabla \phi) & =0 ; \\
\rho(|\nabla \phi|) & =\rho_{0}+\frac{1}{2}\left(v_{\infty}^{2}-|\nabla \phi|^{2}\right) \\
\hat{n} \cdot \nabla \phi & =0 \quad \text { on the disk } \\
\phi & =v_{\infty} x \quad \text { at infinity }
\end{aligned}
$$


here $\hat{n}$ is normal to the disk perimeter.

Rewriting the equation (4) in the frame reference defined by the pole of the disk as the origin, one gets:

$$
\partial_{v}(\rho(v) v) \partial_{x x} \phi+\rho(v) \partial_{y y} \phi=0
$$

where $x$ is the local coordinate tangent to the main flow, and $y$ the orthogonal one. At low velocities this second order partial differential equation is elliptic. It is also possible to show, via an hodograph transformation, that the maximum local speed for a flow around an obstacle, occurs on the boundary of the obstacle. This is in some sense a nonlinear (but still elliptic) generalization of the min-max theorem by Riemann and Liouville. Equation (2) becomes hyperbolic beyond a critical velocity. This happens when $\partial_{v}(\rho(v) v)$ vanishest, that is when the mass current takes its largest possible value for some condition at infinite.

The above criteria $\left(\partial_{v}(\rho(v) v)=0\right)$ gives, for the present model, a critical velocity $v_{c}$ such that: $v_{c}^{2}=\frac{2}{3} \rho_{0}+\frac{1}{3} v_{\infty}^{2}$. When $v_{\infty}$ increases the property of ellipticity of equation (2) is broken first at the pole of the disk, leading to the nucleation of two vortices, one at each pole. As time goes on these vortices are convected downstream by the mean flow. These vortices, once released, induce as well a counterflow because of the circulation condition and this counterflow reduces the velocity on the surface of the disk. This brings back the local velocity at the pole of the disk below the critical speed, and restores there the ellipticity of the equation for the velocity potential. But the vortex is pulled farther and farther downstream, and the counter streaming effect diminishes, till the velocity at the pole reaches eventually the critical value, the conditions at infinity being kept constant; then new vortices are emitted, etc. This describes a more or less periodic release of vortices from the obstacle [3]. We shall study in this article the process of nucleation, namely the way in which a vortex is emitted from the boundary when the local velocity changes slowly from

\footnotetext{
${ }^{4}$ This equation becomes hyperbolic also when $\rho(v)$ vanishes, however, this happens for a larger value of the speed with the present equation of state (relation between pressure and density).
} 
below critical to above critical speed. For this we developpe a phase-dynamics approach for the longwave asymptotics (distances larger than $\xi_{0}$ ).

\section{THE EULER-TRICOMI EQUATION NEAR THE TRANSONIC REGION.}

Suppose that the local velocity at the pole of the disk, $v_{0}$, is close from $v_{c}$; taking this point the origin of the axis, the $x$-axis being tangential to the disk, and the $y$-axis perpendicular. One writes the phase near the pole as $\phi=v_{0} x+\frac{v_{c}}{3} \chi, \chi$ being small; this gives for equation (田), together with (5) (after an elementary redefinition of variables):

$$
-\left(\epsilon+\partial_{x} \chi\right) \partial_{x x} \chi+\partial_{y y} \chi=0
$$

with $\epsilon \sim\left(v_{0}-v_{c}\right) / v_{c}$. The boundary condition (6) becomes:

$$
(x / R, 1) \cdot\left(v_{0}+\frac{v_{c}}{3} \partial_{x} \chi, \frac{v_{c}}{3} \partial_{y} \chi\right)=0 \quad \text { at } \quad y=-\frac{x^{2}}{2 R}
$$

where $(a, b) \cdot\left(a^{\prime}, b^{\prime}\right)=a a^{\prime}+b b^{\prime}$. Neglecting $\partial_{x} \chi$ in the boundary conditions gives:

$$
\partial_{y} \chi=-M \frac{x}{R} \quad \text { at } \quad y=-\frac{x^{2}}{2 R}
$$

With $M=\frac{3 v_{0}}{v_{c}}$, a constant proportional to the actual Mach number. Let us also notice that the boundary condition arises on $y=-\frac{x^{2}}{2 R}$, which is a parabolic approximation of the disk near the pole.

Equation (9) derives from a variational principle with an energy

$$
E=\int d x d y\left[-\frac{1}{6}\left(\epsilon+\partial_{x} \chi\right)^{3}+\frac{1}{2}\left(\partial_{y} \chi\right)^{2}\right]
$$

The solution of (9) satisfying the equation and the boundary condition is

$$
\chi_{0}=-M \frac{x y}{R}
$$

\footnotetext{
${ }^{5}$ This is a direct consequence from equation (4) which is the extremum of $E=\frac{1}{2} \int d x d y \rho(v)^{2}=$ $\frac{1}{2} \int d x d y\left[\rho_{0}+\frac{1}{2}\left(v_{\infty}^{2}-(\nabla \phi)^{2}\right)\right]^{2}$.
} 
however, this one is not sufficient to determine the complete flow in particular the transition to supersonic flow, it is necessary to go up to next order. Writing $\chi=\chi_{0}+\varphi$, one finds:

$$
-\left(\epsilon-M \frac{y}{R}\right) \partial_{x x} \varphi+\partial_{y y} \varphi=0
$$

with

$$
\partial_{y} \varphi=-M \frac{x^{3}}{R^{3}} ; \quad \text { at } \quad y=0
$$

The resulting equation (12) for $\varphi$ is Euler-Tricomil with the boundary condition (13).

The Euler-Tricomi equation may be interpreted as follows: $-\left(\epsilon-M \frac{y}{R}\right)$ represents a generic tangential velocity profile of an ideal flow near a body, since the local main speed diminishes as $y$ increases, that is as one moves far-away from the obstacle. The Mach number is exactly one at $y=\epsilon \frac{R}{M}$. In this equation we have neglected the nonlinear term $\partial_{x} \varphi \partial_{x x} \varphi$ besides $y \partial_{x x} \varphi$ in (12); on the other hand the border of the obstacle has been taken at $y=0$. (The curvature of the obstacle brings a correction to the boundary condition for the dominant order solution, that transforms itself to an extra term in the partial differential equation for the perturbation with a flat boundary $\varphi$.) This assumption is consistent to the following scaling in $\epsilon$ for the coordinates

$$
x \sim \frac{R \epsilon^{3 / 2}}{M} \quad, \quad y \sim \frac{R \epsilon}{M} \quad \text { and } \quad \varphi \sim \frac{R \epsilon^{11 / 2}}{M^{3}} .
$$

Once again, there exists a particular solution of equation (12) satisfying the boundary conditions, so that we will have to focus on the homogeneous solution of its equation. It happens that, $\phi_{0}$, defined as:

$$
\phi_{0}=-M \frac{x^{3} y}{R^{3}}-\epsilon M \frac{x y^{3}}{R^{3}}+M^{2} \frac{x y^{4}}{2 R^{4}}
$$

\footnotetext{
${ }^{6}$ Note that here the variables are in the physical space and not the hodograph variables as in [6]. With this procedure we have considerate directly the boundary conditions, something difficult to work with in the hodograph plane.
} 
satisfies equation (12) with the (13) boundary conditions.

At this point, one can iterate the linear Euler-Tricomi equation by the same procedure, that is by considering how $\phi_{0}$ modifies the true boundary condition and then we compute the next term $\phi_{1}$, and so on. It is then possible to find a polynomial expansion satisfying the full boundary condition. However, the convergence of this expansion is not obvious. This is not an objection in principle because we are looking only local solutions, however we have in mind an outer asymptotics which will mtach with a vortex kind solution (see section VII.

\section{SPECIAL SOLUTIONS OF THE EULER-TRICOMI EQUATION}

Let us first make an "aparte" by looking at some special solutions of equation (12) that might give some idea of what arises when crossing the critical velocity, that is at the transonic transition.

The roots $z(x, y)$ of the cubic polynomial (as well as any linear combination of these three roots):

$$
z^{3}+3\left(\frac{M}{R}\right)^{1 / 3}\left(y-\epsilon \frac{R}{M}\right) z+3 x
$$

are exact solutions of equation (12). Note that for $y>\epsilon \frac{R}{M}$, the cubic polynomial has only

one real root for all values of $x$, whereas, for $y<\epsilon \frac{R}{M}$ there are three real roots inside a semi-cubic parabola defined by $|x| \leq 2 / 3(\epsilon-y)^{3 / 2}$.

This multivaluation of the real roots of the cubic equation (15) means that it is not possible, generally, to follow continuously a root of (15) along a closed path around the origin (see figure (2)). More precisely, such solution will admit a discontinuity in the region inside the semi-cubic. Unless one can regularize the discontinuity which arise along the region multivalued (in the same sense that we have to regularize the over turning of waves dynamics, solutions of nonlinear and non dispersive wave equations), there is no hope of having a stationary solution of our problem except if the discontinuity gap is $2 \pi$. In this case (jump of $2 \pi$ of the phase), we will see that even if both the quantum pressure and the 
limited transonic region have a tendency to restore the smoothness of the solution, there is a critical velocity above which there is no more possibility of having stationary solutions. The way we will treat the equation will hide the multivalued property that we pointed out because we will look on regular stationary solutions (which exist as well as no discontinuity appears), so that the discontinuities will be solved via the general time dependent nonlinear problem.

A general solution arises whenever one consider a continuous superposition in the neutral translation mode of the above solution (15):

$$
\phi(x, y)=\int a_{\xi} z(y, x-\xi) d \xi
$$

equivalent after a change of variable to the integral expresion in $\S 118$ of [6]. This general expression is valid only for $y>\epsilon \frac{R}{M}$ (as well as the roots of the polynomial (15)) where such a change of variable remains well defined.

Before finish this section, let us mention, that another family of solution appears when one takes the Fourier transform of the $x$ variable. Then one has that

$$
\phi_{\nu}(x, y)=\Phi(y) e^{i \nu x}
$$

with $\Phi(y)$ is the Airy function, the detailed analysis of this kind ok solution is elaborated below in a more general way.

\section{REGULARIZATION OF THE SHOCK SOLUTIONS OF THE EULER-TRICOMI EQUATION BY THE QUANTUM PRESSURE.}

As soon as the solutions of the Euler-Tricomi equation appears to be sharper and sharper that the quantum pressure term in the Bernoulli equation (3) is no longuer negligible because it involves higher order derivatives. From the full Bernoulli equation one gets the value of $\rho$ by an implicit relation (we shall consider here also the role of non stationary dependence of phase and density in order to capture the full short wavelength dynamics):

$$
\rho=\rho_{0}+\frac{1}{2}\left(v_{\infty}^{2}-(\nabla \phi)^{2}\right)-\partial_{t} \phi+\frac{1}{4 \rho}\left(\Delta \rho-\frac{(\nabla \rho)^{2}}{2 \rho}\right),
$$


reminding that $\phi=v_{0} \cdot x+\frac{v_{c}}{3}\left(\chi_{0}+\phi_{0}+\varphi\right) \phi$ and $\varphi$ being function of both time and position whereas $v_{0}, \chi_{0}$ and $\phi_{0}$ are independant of time. One can then estimate the value of the quantum pressure at the first order of perturbation, taking $\rho=\rho_{0}+\frac{1}{2}\left(v_{\infty}^{2}-(\nabla \phi)^{2}\right)-\frac{v_{c}}{3} \partial_{t} \varphi$ (it gives then the first non-zero contribution of the quantum pressure); coupling this with the (now) non-stationnary mass conservation equation and restoring the first nonlinear terms as well as the constant terms gives for the phase equation:

$$
-\left(\epsilon-M \frac{y}{R}\right) \partial_{x x} \varphi+\partial_{y y} \varphi-\xi_{0}^{2} \partial_{x^{4}} \varphi=\frac{1}{v_{c}^{2}} \partial_{t t} \varphi+\frac{M}{v_{c}} \partial_{t x} \varphi+\partial_{x} \varphi \partial_{x x} \varphi+\partial_{x} \phi_{0} \partial_{x x} \varphi+\partial_{x x} \phi_{0} \partial_{x} \varphi+\partial_{x} \phi_{0} \partial_{x x} \phi_{0}
$$

We have kept in this equation only the most important term of each contribution. The quantum pressure term, $\xi_{0}^{2} \partial_{x^{4}} \varphi$ should be multiplied by a number that we have taken to one by simplicity.

The way this equation (16) is written is dictated by our method of resolution: the left hand side will be in fact treated as the main equation, linear and homogeneous, while the right hand side corresponding to perturbations which will be incorporated terms by terms. As said before the boundary conditions are taken homogeneous:

$$
\partial_{y} \varphi=0 \quad \text { at } \quad y=0 .
$$

Let us study the regularisation of the solution above the threshold, first by adding the quantum pressure then by considering cross terms involving $\varphi$ and $\phi_{0}$; at these points the analysis remains linear so that we will just be able to look to homogeneous solutions of our problem, without solving for the amplitude; finally we will focus on the global nonlinear problem which allows to calculate amplitude equations and leads to the study of the time-dependent evolution.

First we look at the left hand side of equation (16), taking the right hand side as zero. The boundary conditions are $\partial_{y} \varphi=0$ at $y=0$, so that one can expand the solution as a linear superposition of functions. The equation can be solved as the Euler-Tricomi one, using the Airy function. 
We seek solutions of the form $\varphi_{\nu}=e^{ \pm i \nu x} \zeta(y), \zeta(y)$ satisfying the Airy equation:

$$
\zeta^{\prime \prime}+\nu^{2}\left(\epsilon-\xi_{0}^{2} \nu^{2}-M \frac{y}{R}\right) \zeta=0
$$

which non-divergent solution is known to be the Airy function $\Phi(\cdot)$; therefore the solution reads:

$$
\varphi_{\nu}=A \cdot e^{i \nu x} \Phi\left[\left(\frac{\nu^{2} M}{R}\right)^{\frac{1}{3}}\left(y-\frac{R}{M}\left(\epsilon-\nu^{2} \xi_{0}^{2}\right)\right)\right] .
$$

being $A$ a complex amplitude fixed by nonlinearities at next order. As the function $\Phi(s)$ does not possess extrema for $s>0$ the boundary condition might be satisfied for $\epsilon>0$ only, otherwise $A=0$. Let $s_{n}$ be the $n^{t h}$ zero of $\Phi^{\prime}(s)$ then the only possible values for the wavenumber $\nu$ are such that they satisfy a "quantization condition" for a given $\epsilon$ :

$$
\epsilon=\left(\frac{M \xi_{0}}{R}\right)^{\frac{2}{3}}\left(-s_{n}\right)\left(\xi_{0} \nu\right)^{\frac{2}{3}}+\left(\xi_{0} \nu\right)^{2}
$$

which have been represented on figure 3. So, if $\epsilon$ is less than a critical value, one can observe that, because of the quantum pressure, the homogeneous Euler-Tricomi equation has just the null function as solution $(A=0)$. This critical value (the minimum of the curve plotted in figure (3) is easy to evaluate:

$$
\epsilon_{c}=4 \times\left(-\frac{s_{1}}{3}\right)^{\frac{3}{4}} \times \sqrt{\frac{M \xi_{0}}{R}}=4 \xi_{0}^{2} \nu_{c}^{2} \quad \text { with } \quad \nu_{c}=\left(-\frac{s_{1}}{3}\right)^{\frac{3}{8}} \times\left(\frac{M}{R \xi_{0}^{3}}\right)^{\frac{1}{4}}
$$

the critical wave number. Generically, this means that for $\epsilon<\epsilon_{c}$ the stationary solution $\left(\phi_{0}\right)$ might describe the dynamics at the order of the Taylor expansion whereas for $\epsilon \geq \epsilon_{c}$ the amplitude of a solution for $\nu=\nu_{c}$ can expand. At this point, the amplitude cannot be known and has to be found as the result of the nonlinear and time dependent analysis. It follows now a general scheme, which consists of the evaluation of the amplitude and the corrections of the general solution by writing a solvability condition for their existence.

We seek a solution of the form:

$$
\phi=A(x) \cdot e^{ \pm i \nu_{c} x} \Phi\left[\left(\frac{\nu_{c}^{2} M}{R}\right)^{\frac{1}{3}}\left(y-\frac{3 R \epsilon_{c}}{4 M}\right)\right]+\varphi_{1}(y) e^{ \pm i \nu_{c} x} .
$$


Now $A(x)$ is a slightly varying amplitude on the horizontal variable $x$ (the time dependance will be taken account in the next section) $\varphi_{1}$ being a small correction to $\varphi$ depending on $y$ only (the $x$ dependance coming from the main term $e^{i \nu_{c} x}$ ). This first correction of our equation (16) will takes in account the cross term between $\varphi$ and $\phi_{0}$; the slow behavior of $A(x)$ on $x$ requires:

$$
\left|\frac{\partial_{x} A(x)}{A(x)}\right| \ll \nu_{c} .
$$

In addition let us define the $\zeta(y)$, the Airy function that we use: $\zeta(y)=\Phi\left(\left(\frac{\nu_{c}^{2} M}{R}\right)^{\frac{1}{3}}(y-\right.$ $\left.\frac{3 R \epsilon_{c}}{4 M}\right)$ ). The cross contribution to the nonlinear term $\phi_{x} \phi_{x x}$ which we have neglected until now, give two terms linear in $\varphi$ in equation (16): $\partial_{x} \phi_{0} \partial_{x x} \varphi$ and $\partial_{x x} \phi_{0} \partial_{x} \varphi$.

Therefore we obtain for the phase the following equation:

$$
\begin{aligned}
\mathcal{L}_{0} \varphi_{1}= & {\left[\nu_{c}^{2}\left(-\left(\epsilon-\epsilon_{c}\right)-\partial_{x} \phi_{0}\right) A(x)+i \nu_{c} \partial_{x x} \phi_{0} A(x)+2 i \nu_{c}\left(\frac{\epsilon_{c}}{2}-M \frac{y}{R}+\partial_{x} \phi_{0}\right) A^{\prime}(x)\right.} \\
& \left.+\partial_{x x} \phi_{0} A^{\prime}(x)-\left(\frac{\epsilon_{c}}{2}+M \frac{y}{R}-\partial_{x} \phi_{0}\right) A^{\prime \prime}(x)\right] \zeta(y)
\end{aligned}
$$

where $\mathcal{L}_{0}$ is the linear operator acting on the one variable function space:

$$
\mathcal{L}_{0}=\partial_{y y}+\nu_{c}^{2}\left(3 \xi_{0}^{2} \nu_{c}^{2}-M \frac{y}{R}\right)
$$

Notice that $\zeta(y)$ is in the kernel of $\mathcal{L}_{0}$. The solvability condition says that the right hand side of (18) is orthogonal to members of the kernel of $\mathcal{L}_{0}$ joint operator. With the scalar product $\langle f, g\rangle=\int_{0}^{\infty} f(y) g(y) d y$ the linear operator $\mathcal{L}_{0}$ is self-adjoint, therefore $\zeta(y)$ belongs to its kernel. The solvability condition of (18) gives an equation for the slowly varying amplitude (keeping the first order in $A$ only) $A(x)$ :

$$
A^{\prime \prime}(x)+\left(\frac{\epsilon-\epsilon_{c}}{4 \xi_{0}^{2}}-\frac{3 \nu_{c}^{2} x^{2}}{2 R^{2}}\right) A(x)=0 .
$$

One recognize the equation of the quantum harmonic oscillator; $\frac{\epsilon-\epsilon_{c}}{4 \xi_{0}^{2}}$ being the equivalent of the energy. It has non zero solution exists if $\epsilon>\epsilon_{c}^{\prime}$ only. The lowest value of epsilon with non-zero solution satisfies:

$$
\epsilon_{c}^{\prime}-\epsilon_{c}=\sqrt{6 \epsilon_{c}} \frac{\xi_{0}}{R} \ll \epsilon_{c}
$$


Note that this correction is small with respect to the first one. The corresponding solution at $\epsilon=\epsilon_{c}^{\prime}$ reads

$$
A(x)=A e^{-\frac{\left(\epsilon_{c}^{\prime}-\epsilon_{c}\right) x^{2}}{8 \xi_{0}^{2}}}=e^{-\frac{x^{2}}{2 l^{2}}}
$$

being $l$ the characteristic length of $A(x), l=2 \sqrt{\xi_{0} R} /\left(6 \epsilon_{c}\right)^{\frac{1}{4}}$ (note that this agrees with the condition of validity of this WKB approach $\nu_{c} l \gg 1$ ). As for the former treatment, a non zero amplitude $A$ can arise only if $\epsilon>\epsilon_{c}^{\prime}$; this amplitude and its dynamics will be obtained by the time dependent nonlinear system for $\epsilon \sim \epsilon_{c}^{\prime}$.

\section{AMPLITUDE EQUATION FOR THE SADDLE-NODE BIFURCATION}

In this section we shall look at the general spatio-temporal dependence of the solution of (16) for $\epsilon \sim \epsilon_{c}^{\prime}$; writing now the Ansatz for the phase $\varphi$ as $(A(t)=\alpha(t)+i \beta(t))$ :

$$
\varphi=\left(\alpha(t) \sin \left(\nu_{c} x\right)+\beta(t) \cos \left(\nu_{c} x\right)\right) e^{-\frac{x^{2}}{2 l^{2}}} \zeta(y)+\varphi_{1}(y) \cos \left(\nu_{c} x+\Omega\right)+\varphi_{2}(x) \zeta(y)
$$

being $\varphi_{1}(y)$ and $\varphi_{2}(x)$ small corrections to $\varphi$. Imposing a solvability condition (one first along $y$, then along $x$ ), one gets the following dynamical system:

$$
\begin{aligned}
& \frac{1}{v_{c}^{2}} \alpha^{\prime \prime}(t)-M \frac{\nu_{c}}{v_{c}} \beta^{\prime}(t)=\nu_{c}^{2}\left(\epsilon-\epsilon_{c}^{\prime}\right) \alpha(t)+\delta e^{-\frac{\nu_{c}^{2} l^{2}}{2}}+\nu_{c}^{3}\left(\alpha^{2}(t)-\beta^{2}(t)\right) e^{-\frac{\nu_{c}^{2} l^{2}}{6}} \\
& \frac{1}{v_{c}^{2}} \beta^{\prime \prime}(t)+M \frac{\nu_{c}}{v_{c}} \alpha^{\prime}(t)=\nu_{c}^{2}\left(\epsilon-\epsilon_{c}^{\prime}\right) \beta(t)+2 \nu_{c}^{3} \alpha(t) \beta(t) e^{-\frac{\nu_{c}^{2} l^{2}}{6}}
\end{aligned}
$$

with $\delta \sim \xi_{0} / R^{2}$. For convenience, and because it does not change the meaning of the dynamics, we have taken the ratio between the different constant coming from solvability integration equal to one. The transcendental terms (in $e^{-\nu_{c}^{2} l^{2}}$ ) are due to the interaction between the nonlinear term and the constant term $\partial_{x} \phi_{0} \partial_{x x} \phi_{0}$ with the modes $\sin \left(\nu_{c} x\right) e^{-\frac{x^{2}}{2 l^{2}}}$ and $\cos \left(\nu_{c} x\right) e^{-\frac{x^{2}}{2 l^{2}}}$.

The system (19) could be written in a single complex equation for the complex amplitude (after an appropiate change of variable and rescaling): 


$$
Z_{t t}+i \omega Z_{t}=\mu+Z^{2}
$$

Here $\mu=-\left(\epsilon-\epsilon_{c}^{\prime}\right)^{2} e^{\frac{\nu_{c}^{2} l^{2}}{3}} / 4 \nu_{c}^{2}+\delta e^{-\frac{\nu_{c}^{2} l^{2}}{3}} / \nu_{c}^{3}$.

The stationary solutions are $Z_{ \pm}= \pm \sqrt{-\mu}$ that is for negatives values of $\mu$ one preserves an odd symmetry of $\varphi(x, y)$ along the $x$-axis, this symmetry is broken as soon as $\mu$ changes sign and the further evolution is more complex.

In terms of the physical parameters the region $\mu<0$ is for $\left(\epsilon-\epsilon_{c}^{\prime}\right)^{2}>\frac{4 \delta}{\nu_{c}} e^{-\frac{2 \nu_{c}^{2} l^{2}}{3}}$, leading to a new critical velocity $\epsilon_{t}$, defined as:

$$
\epsilon_{t}=\epsilon_{c}^{\prime}-2 \sqrt{\frac{\delta}{\nu_{c}}} e^{-\frac{\nu_{c}^{2} l^{2}}{3}} .
$$

Notice that for small aspect ratio, the main correction for the critical velocity is in $\sqrt{\frac{\xi_{0}}{R}}$ and is determined by $\epsilon_{c}$ whereas $\epsilon_{c}^{\prime}$ and $\epsilon_{t}$ induce just exponentially small corrections.

Figure (4) shows the real roots of $Z_{ \pm}$as a function of $\mu$ or better as a function of $\epsilon-\epsilon_{t}$. One can identify two branches: one for low velocities $\left(\epsilon<\epsilon_{t}\right)$; the other one for high velocities. As we have conducted our calculations, starting from stationary solution for low velocity and then adding the nonlinear dynamics step by step, this second branch for high velocity is irrelevant in our problem. As shown on figure (业, for $\epsilon<\epsilon_{t}$ there are two roots of the stationary equation, roots that greather for $\epsilon=\epsilon_{t}$, whereas there is no more roots for $\epsilon$ greater than $\epsilon_{t}$, giving rise to a saddle-node bifurcation. The linear stability analysis of the stationary solutions $Z_{ \pm}$gives the following dynamic $\left(Z(t)=Z_{ \pm}+z(t)\right.$, and $\left.|z(t)| \ll\left|Z_{ \pm}\right|\right)$:

$$
z_{t t}+i \omega z_{t}= \pm 2 \sqrt{-\mu} z
$$

Therefore, one notes that for:

$$
\epsilon<\epsilon_{c}^{\prime}-\sqrt{\frac{M^{4}}{16}+4 \frac{\delta}{\nu_{c}} e^{-\frac{2}{3} \nu_{c}^{2} l^{2}}}
$$

one branch is stable $\left(Z_{-}\right.$, representing by $A_{-}$on figure (4) $)$and the other is always unstable as in ordinary saddle-node bifurcations. However, because of to the oscillatory term $i \omega z_{t}$, when approaching the bifurcation, both solutions are stable. This happens in the range of 
values of $\epsilon$ such a that

$$
\epsilon_{c}^{\prime}-\sqrt{\frac{M^{4}}{16}+4 \frac{\delta}{\nu_{c}} e^{-\frac{2}{3} \nu_{c}^{2} l^{2}}}<\epsilon<\epsilon_{t}
$$

The resulting phase diagram of the bifurcation has been plotted on figure (5). We argue that this saddle-node bifurcation gives a consistant scenario of the vortex nucleation seen in figure (11). They appear as a consequence of the disappearence of stationnary solutions on the phase dynamic approch. The vortex diminishes the local velocity so that the flow comes back to a description valid for $\epsilon<\epsilon_{t}$ and when the vortex is far enough, because of the advection due to the mean velocity, $\epsilon$ pass again through the transition and we got a periodic vortex-nucleation process. The saddle-node bifurcation appears in our case to be richer because close to the transition both stationnary solutions are stable. This result is in a good agreement with numerical solution by Huepe and Brachet [8].

\section{BREAKDOWN OF THE PHASE DESCRIPTION, THE APPEARANCE OF VORTEX MOTION, CONCLUSIONS AND FURTHER MISCELLANEOUS}

We have in the former section reviewed the phase description, that explains the disappearence of any stationary solution for the flow problem. This is the first step toward the nucleation of a vortex. Briefly, one can retain that this periodic behavior appears as a saddle-node bifurcation where two branches of stationnary solutions collapse giving rise for larger velocities to non stationnary solution at all. The numerous critical parameters we have mentionned might be simplified if one see that as $R \gg \xi_{0}$, we have $\epsilon_{t} \sim \epsilon_{c}^{\prime} \sim \epsilon_{c}$. This allows to claim that the critical velocity for vortex nucleation $v_{v}$ reads at first order correction of the critical speed:

$$
v_{v}-v_{c} \propto \sqrt{\frac{\xi_{0}}{R}}+\mathcal{O}\left(e^{-\nu_{c}^{2} l^{2}}\right) .
$$

where $v_{c}$ is the Landau critical velocity.

Finally, it remains to match the vortex nucleation as a process being part of the same

evolution of the dynamical saddle-node bifurcation. For simplicity we will consider the 
amplitude equation (20) where we have dropped the first order time derivative, a term much smaller than the other ones as one approaches the time when nucleation occurs (see the scaling below):

$$
\ddot{Z}(t)=Z^{2}(t)+\mu
$$

where $\mu$ is related to $\epsilon-\epsilon_{t}$. When $\mu$ is negative, there is two real stationnary solutions $(Z=$ $-\sqrt{-\mu}$ the stable, $z=\sqrt{-\mu}$ the unstable) corresponding to $\alpha_{ \pm}$. The saddle-node bifurcation is crossed when $\mu$ becomes positive. This can be studied by taking $\mu=t$ (by rescaling, no multiplying factor is needed) and then we obtain the first Painlevé transcendent $[$ [9]:

$$
\ddot{Z}=Z^{2}+t
$$

A convenient change of variable, for $t<0$ is:

$$
z=\sqrt{-t} W(T) ; \quad T=\frac{4}{5}(-t)^{5 / 4}
$$

and gives the following equation:

$$
\ddot{W}+\frac{\dot{W}}{T}-\frac{4}{25} \frac{W}{T^{2}}=W^{2}-1
$$

${ }^{7}$ One may note that taking $\mu=t$ means to take $\epsilon=\dot{\epsilon} t$ in equation (16). This gives

$$
-\left(\dot{\epsilon} t-M \frac{y}{R}\right) \partial_{x x} \varphi+\partial_{y y} \varphi-\frac{1}{v_{c}^{2}} \partial_{t t} \varphi-\frac{M}{v_{c}} \partial_{t x} \varphi=0
$$

which, after a change of variables of the form: $\eta=\dot{\epsilon} t-M y / R$ and $\zeta=M v_{0} t / R+\dot{\epsilon} y / v_{0}$, and neglecting the linear derivative in time as in (21), leads to:

$$
-\eta \partial_{x x} \varphi+\left(M^{2} / R^{2}-\dot{\epsilon}^{2} / v_{0}^{2}\right)\left(\partial_{\eta \eta} \varphi-\partial_{\zeta \zeta} \varphi\right)=0
$$

A kind of Euler-Tricomi equation, interesting by itself. One note that, if the rate of acceleration at infinity $\dot{\epsilon}$ is larger than $M v_{0} / R$, the nucleation process is caused by a dynamical instability not by a sonic transition as is described in this article. 
It typically gives the two stationnary solutions $W= \pm 1$ for large $T$ and it can be solved in terms of Weierstrass function for large $T$ [9]. Equation (21) is known for giving finitetime singularities which shows that the slowly varying approximation of the phase dynamics breaks at some point. In addition, one can notice that $Z$ is related to the velocity of the fluid in the moving frame (of velocity $v_{0}$ ). Then, one expect to relate the amplitude $Z$ to a order parameter which parametrizes a continuous family of solutions of the full nonlinear Schödinger equation (11). Jones and Roberts [10] found the kind of solution that we are interested, in two and three spatial dimensions, consisting in axisymmetric solitary structures. The solution proposed is

$$
\phi=-U x+m \frac{2 x\left(1-U^{2}\right)}{x^{2}+\left(1-U^{2}\right) y^{2}} .
$$

$m$ being a constant and $U$ the free parameter characterizing the whole family of solutions.

Whenever the parameter (a velocity) $U$ of these solutions is close to the unity (the sound speed) one identifies the relative speed $U-1$ (via a change of variable) with the true order parameter $\alpha \& \beta$ of the saddle-node bifurcation.

On the other hand as $U$ goes to zero one matches the solution (22) with a pair of moving vortices, one with negative topological charge located in $(x=0, y=a)$ while the other is an image one inside the $\operatorname{disk}(x=0, y \approx-a)]^{8}$ :

$$
\begin{aligned}
\phi & =-\frac{U x}{\sqrt{1-U^{2}}}+\arctan \left(\frac{(y+a) \sqrt{1-U^{2}}}{x}\right)-\arctan \left(\frac{(y-a) \sqrt{1-U^{2}}}{x}\right) \\
& \approx-\frac{U x}{\sqrt{1-U^{2}}}+\frac{2 x a \sqrt{1-U^{2}}}{x^{2}+\left(1-U^{2}\right) y^{2}}
\end{aligned}
$$

One relates very easily the distance $a$ in (23) with the speed $U$ in (22) by $U=\frac{1}{2 a}$ : the Hemholtz law of motion for point-like vortices. As a diminishes, a vortex pair appears. So in the dynamical context $a$ satisfy formally the same equation than the quantity $z$ of (21) or $\alpha$ of (19), when the time dependant solution evolves (after crossing the saddle-node

\footnotetext{
${ }^{8}$ Note that at first order the dependence on the radii $R$ of the disk is not relevant to this solution.
} 
bifurcation), a vortex appears as $\alpha$ increases, the second vortex of the pair being formally inside the disk in other to preserve the boundary condition.

Finally, it is of a general interest to note that the shallow water equations have exactly the same shape that the set (20,3) in the case of potential fluid motion (see [6]). In fact the shallow water equations are

$$
\begin{aligned}
\partial_{t} h & =-\nabla \cdot(h \mathbf{v}) ; \\
\partial_{t} \mathbf{v}+\mathbf{v} \cdot \nabla \mathbf{v} & =-g \nabla h .
\end{aligned}
$$

After imposing a vortexless flow: $\operatorname{rot} \mathbf{v}=0$, i.e. $\mathbf{v}=\nabla \phi$, and neglecting the quantum pressure term in (3), one identifies the height of fluid $h$ with the superfluid density $\rho$, and the potential fluid velocity with the phase of the condensate wave function. It is possible to get a short scale term in the shallow water equations by adding a capillary term, therefore it exist a complete analogy with the analysis developed in extenso in this article. Perhaps a transonic transition with the predicted scenario is observable in mercury where the kinematic viscosity is very low. However, the final state will be different because the deep significance of the phase of the wave function $\psi$ does not extend the existence of quantized circulation vortices for the case of surface waves, where as it is well known the circulation takes any value. 


\section{REFERENCES}

[1] C. Josserand, Dynamique des Superfluides: Nucleation de vortex et transition de premier ordre, Thesis Université Paris VI (1997).

[2] V.L. Ginzburg and L.P. Pitaevskǐ̌, Sov. Phys. JETP 7, 858 (1958); L.P. Pitaevskiř, Sov. Phys. JETP, 13, 451 (1961); E.P. Gross, J. Math. Phys. 4, 195 (1963).

[3] T. Frisch, Y. Pomeau and S. Rica, Phys. Rev. Letters, 69, 1644 (1992).

[4] Y. Pomeau and S. Rica, Comptes Rendus Acad. Sc. (Paris), t. 316 Série II, 1523 (1993).

[5] V. Hakim, Phys. Rev. E 55, 2835, (1997).

[6] L.D. Landau and E.M. Lifshitz, Fluid Mechanics, Pergamon Press (Oxford) 1987.

[7] L.D. Landau and E.M. Lifshitz, Quantum Mechanics, Pergamon Press (Oxford).

[8] C. Huepe et M.E. Brachet, Comptes Rendus Acad. Sc. (Paris), t. 325 IIb, 195 (1997).

[9] E.L. Ince, in chapter 14 of Ordinary Differential Equations, Dover, (New York) (1956).

[10] C.A. Jones and P.H. Roberts, J. Phys. A: Math. Gen. 15, 2599 (1982). 


\section{FIGURES}

a)

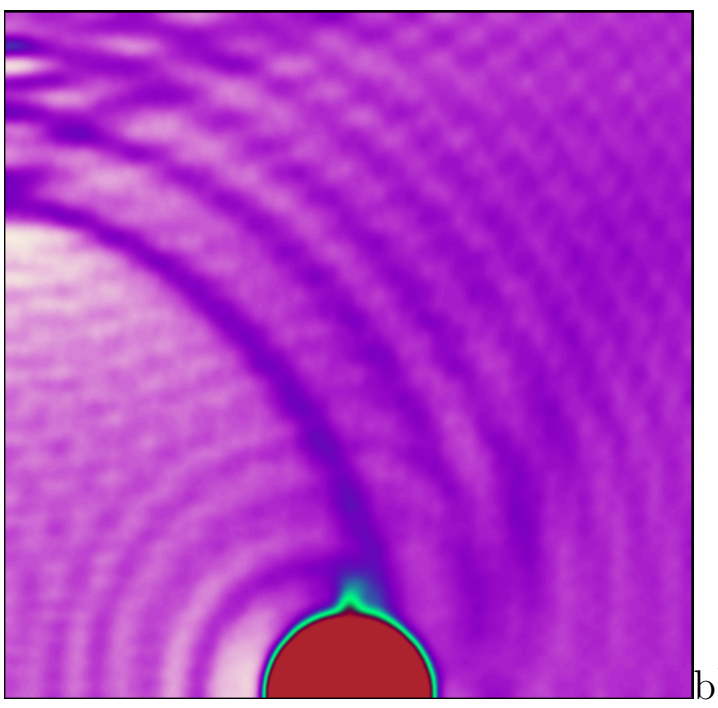

c)

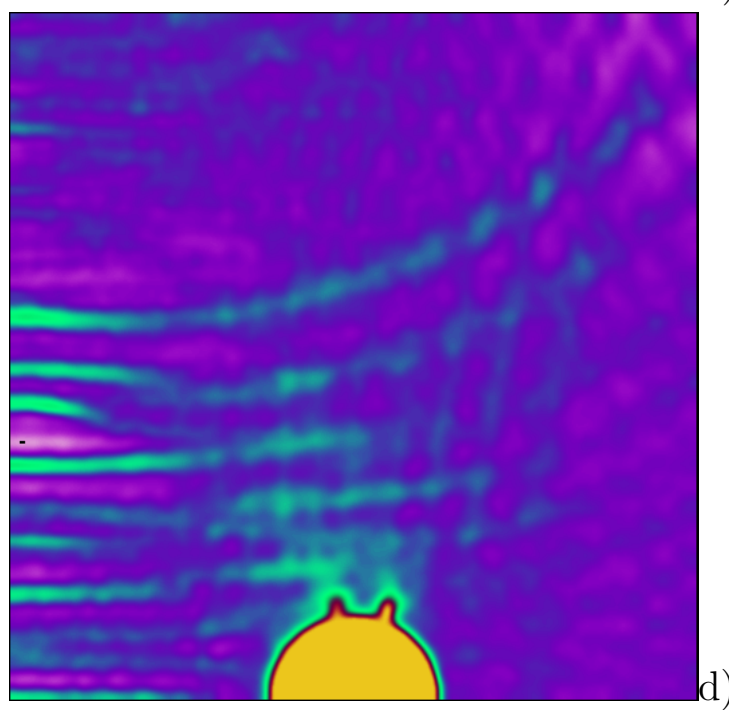

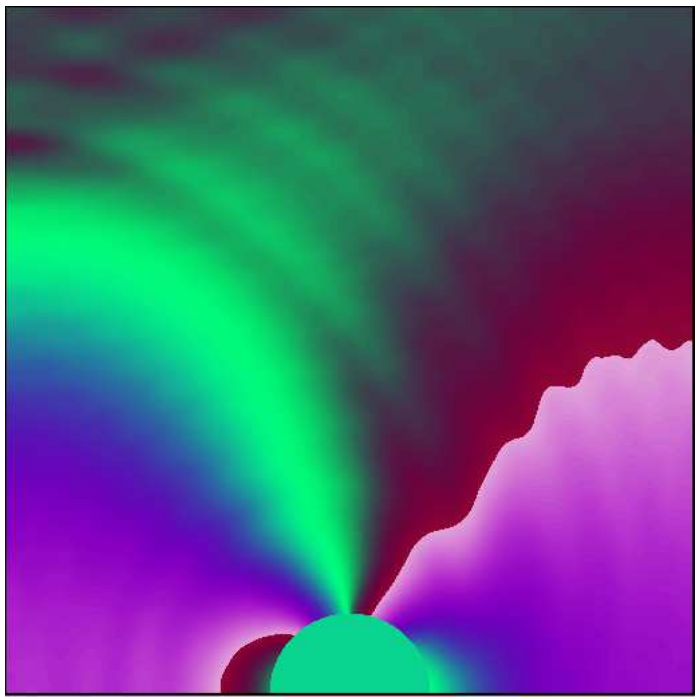

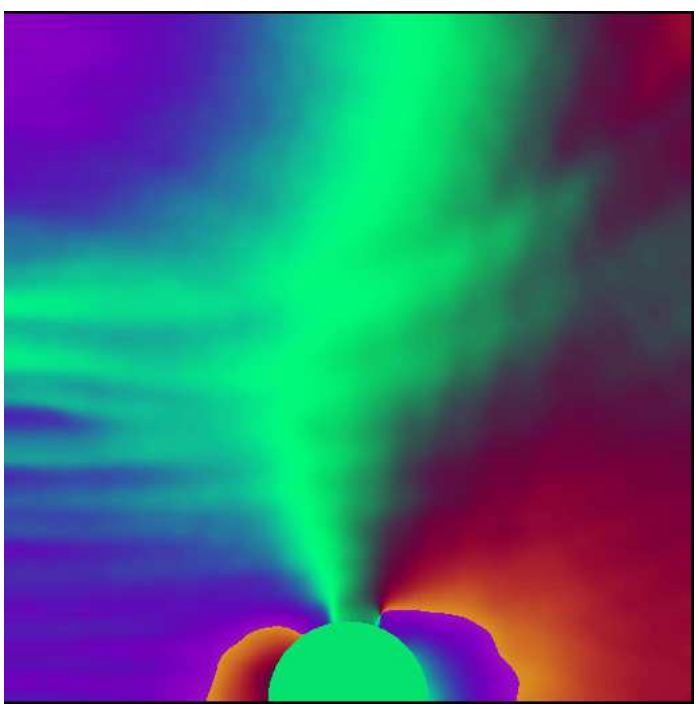

FIG. 1. Numerical simulation of the nonlinear Schrödinger equation for a bidimensional flow around half a disk; the velocity at infinity is $v_{\infty}=0.442$ and we have taken $d x=0.125$ the mesh grid and the radius of the disk is $R=7.5$. a) \& b) respectively the modulus and the phase of the wave function at $t=20$ time unit of NLS. The densite and the phase go up from bright to dark color. One can see the low density around the top of the obstacle, due to a Bernoulli effect. c) \& d) same functions at $t=50.6$. A low density structure is advected by the flow (at right of the top of the disk). One can see a phase discontinuity and the tip of it where the phase is not defined this is the signature of a topological defect, that is a quantized vortex. 


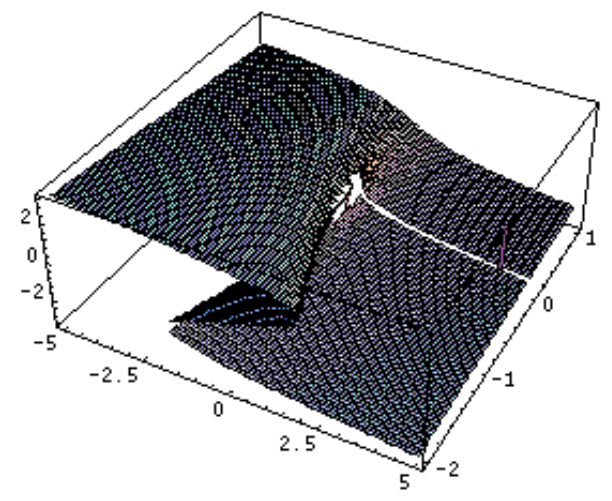

FIG. 2. Shape of $z(x, y)$, for $\epsilon=0$ around the origin.

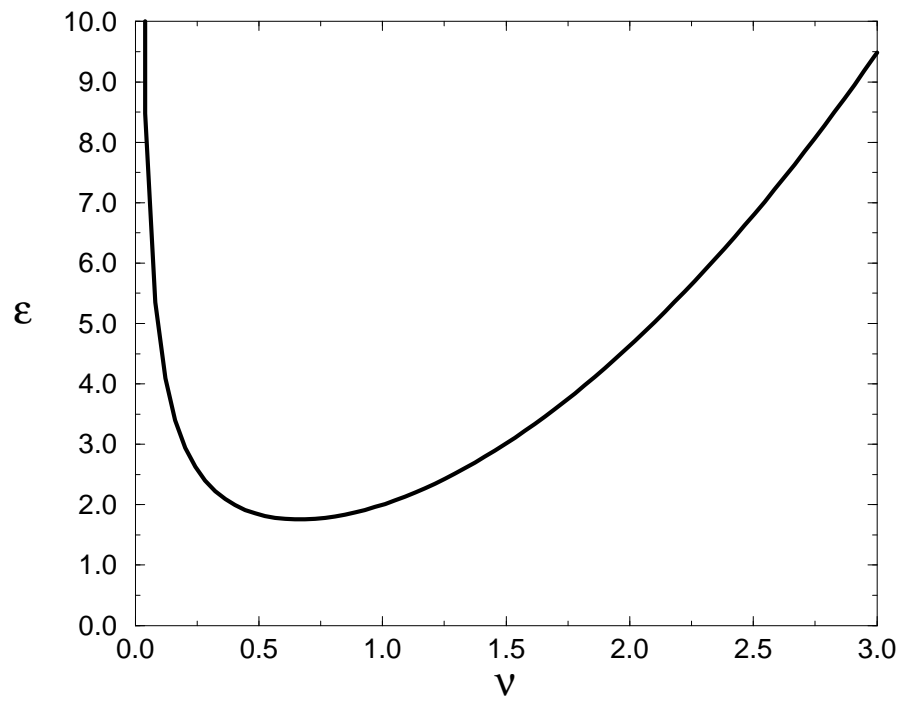

FIG. 3. Relation between $\epsilon$ and $\nu$ the wave number for $R / \xi_{0} \simeq 1$. 


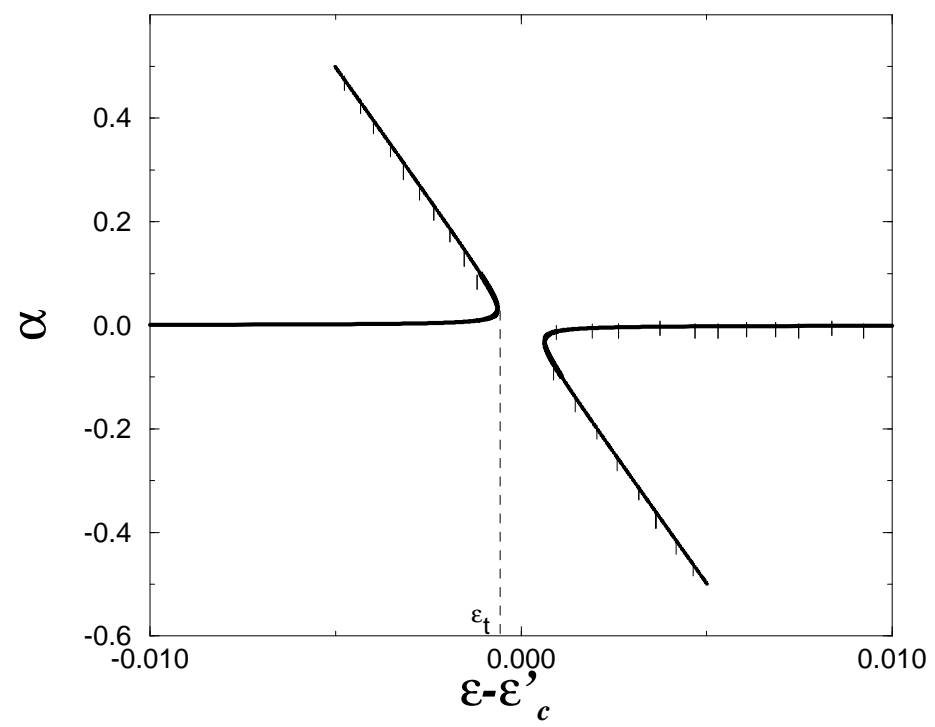

FIG. 4. Stationary amplitude of the phase equation for $\frac{\xi_{0}}{R}=0.1$. The part for $\epsilon>\epsilon_{c}^{\prime}$ is not valid in our approximations whereas we have dashed the unstable solutions.

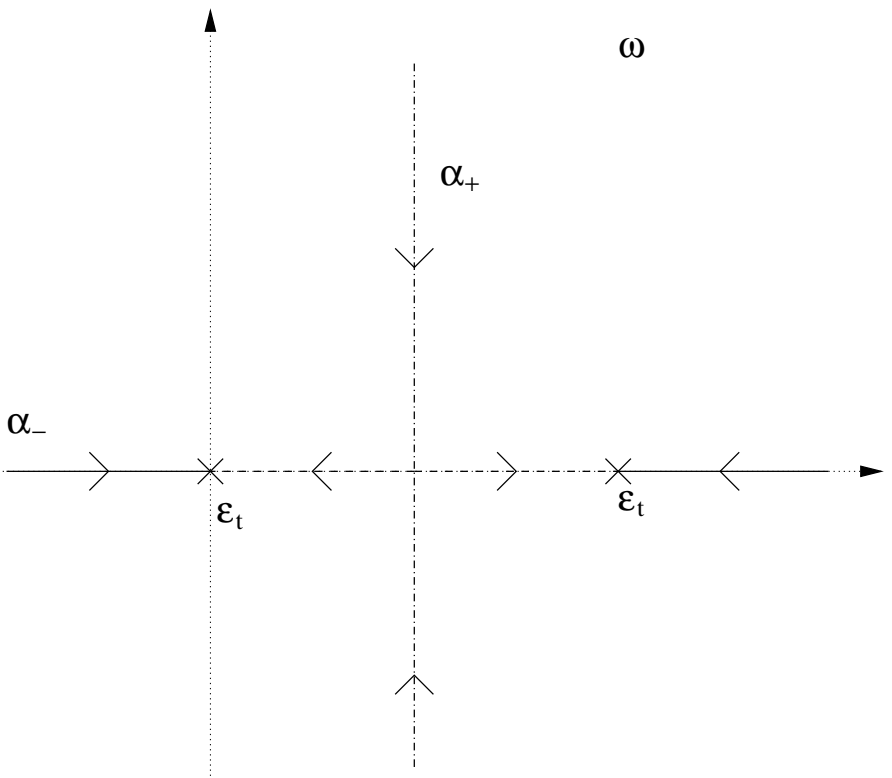

FIG. 5. Stability diagramm of the stationary solutions $\alpha_{ \pm}$in the $\omega$ complex plane (the stability is studied via the expression $w(t)=a \cdot e^{i \omega t}, a$ being an amplitude. The small dashed line corresponds to the real and imaginary axes whereas the large dashed line indicates the stability evolution of $\alpha_{+}$as $\epsilon$ increases untill $\epsilon_{t}$ where the stationnarity disappears as the collapse of $\alpha_{+}$and $\alpha_{-}$. The thick line represents the same evolution for $\alpha_{-}$. 
vortex
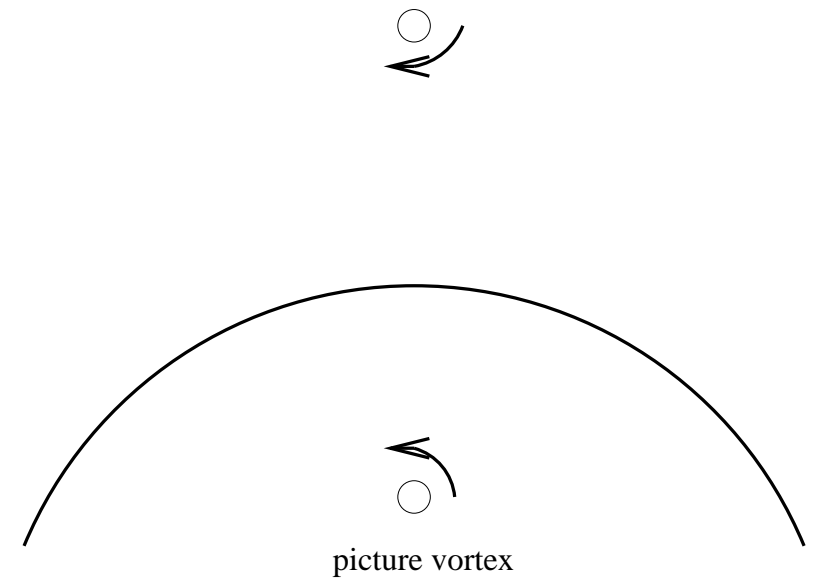

FIG. 6. Position of the vortices for the unstable stationnary solution. 